\title{
Optimization of sparse recovery based on recursive unbiased predictive risk estimate
}

\author{
Feng Xue ${ }^{*}$, Peng Liu, and Jiaqi Liu \\ National Key Laboratory of Science and Technology on Test Physics and Numerical Mathematics, \\ Beijing, 100076, China
}

*Corresponding Author: fxue2012@gmail.com

\begin{abstract}
Sparse recovery via $\ell_{1}$-penalized minimization can be typically solved by iteratively reweighted least squares (IRLS). The reconstruction quality is generally sensitive to the value of regularization parameter. In this wok, we propose a novel data-driven optimization scheme based on minimization of unbiased predictive risk estimate (UPRE). For a given IRLS iterate, we decompose and linearize the update by matrix splitting strategy, which makes the computation of Jacobian matrix tractable; and the UPRE of the IRLS iterate can be recursively evaluated. The developed recursive UPRE enables us to monitor the reconstruction error during the IRLS iterations, which can be applied to optimize regularization parameter. Numerical tests demonstrate the reliability of the recursive UPRE and the optimality of sparse reconstruction.
\end{abstract}

Keywords: Sparse reconstruction, iteratively reweighted least squares (IRLS), unbiased predictive risk estimate (UPRE).

\section{Introduction}

Consider the following standard linear inverse problem: estimate the unknown coefficients $\mathbf{x} \in \mathbb{R}^{N}$ from a linear model $^{(1,2)}$ :

$$
\mathbf{y}=\mathbf{A x}+\epsilon, \quad \mu=\mathbf{A} \mathbf{x}
$$

where $\mathbf{y} \in \mathbb{R}^{M}$ is the observed data, $\mathbf{A} \in \mathbb{R}^{M \times N}$ is a deterministic design matrix, $\epsilon$ is a vector of i.i.d. zero-mean Gaussian random variable with known variance $\sigma^{2}>0$. The classical approaches, e.g. ordinary least squares and ridge regression, generally have the drawbacks in prediction accuracy and interpretation ${ }^{(2)}$. In many applications, e.g. statistical inference ${ }^{(1,2)}$, signal recovery $^{(3,4)}$ and compressed sensing ${ }^{(5)}$, it is preferable to promote the sparsity of $\mathbf{x}$ in some transform domain (e.g. wavelets), which is often formulated as the $\ell_{1}$-penalized linear regression ${ }^{(2,3)}$ :

$$
\text { P: } \quad \widehat{\mathbf{x}}_{\lambda}=\arg \min _{\mathbf{x}} \underbrace{\frac{1}{2}\|\mathbf{y}-\mathbf{A x}\|_{2}^{2}+\lambda\|\mathbf{D} \mathbf{x}\|_{1}}_{\mathcal{L}(\mathbf{x})}
$$

Here, $\mathbf{D} \in \mathbb{R}^{L \times N}$ denotes a decomposition that is believed to have a sparse representation of $\mathbf{x} . \lambda>0$ is a regularization parameter that balances the data fidelity and the sparsity enhancement ${ }^{(1,3)}$.

There is a vast literature dedicated to solving $\mathbf{P}^{(3,4)}$. Iteratively reweighted least squares (IRLS) is an appealing choice due to its superior convergence speed ${ }^{(3,6)}$. We use IRLS to solve $\mathbf{P}$ in this paper. Note that we are not going to develop a more efficient algorithm to solve $\mathbf{P}$, but will focus on the reconstruction quality, i.e., how to optimize the sparse reconstruction $\widehat{\mathbf{x}}_{\lambda}$, solved by a given IRLS iterate.

It has been recognized that $\widehat{\mathbf{x}}_{\lambda}$-the solution to $\mathbf{P}$-is generally sensitive to the value of regularization parameter $\lambda^{(7,8)}$. Hence, many regularized iterative reconstruction algorithms, e.g. IRLS ${ }^{(6)}$ and FISTA $^{(4)}$, often require selection of the appropriate value for $\lambda$. A number of criteria for this selection have been proposed, e.g. discrepancy principle ${ }^{(9)}$, L-curve method ${ }^{(10)}$ and generalized cross validation $(\mathrm{GCV})^{(11)}$.

Squared-error-based criterion can be a promising alternative to others: the prediction accuracy is often quantified in terms of expected prediction error (EPE for short $)^{(1,12)}$

$$
\mathrm{EPE}=\frac{1}{M} \mathbb{E}\left\{\left\|\mu-\widehat{\mu}_{\lambda}\right\|_{2}^{2}\right\}=\frac{1}{M} \mathbb{E}\left\{\left\|\mathbf{A x}-\mathbf{A} \widehat{\mathbf{x}}_{\lambda}\right\|_{2}^{2}\right\}
$$

Since EPE is inaccessible in practice due to unknown $\mu$ and $\mathrm{x}$, the unbiased predictive risk estimate (UPRE) 
provides an unbiased substitute for $\mathrm{EPE}^{(1,12,13)}$ :

$$
\operatorname{UPRE}\left(\widehat{\mu}_{\lambda}\right)=\frac{1}{M}\left\|\mathbf{A} \widehat{\mathbf{x}}_{\lambda}-\mathbf{y}\right\|_{2}^{2}+\frac{2 \sigma^{2}}{M} \operatorname{Tr}\left(\mathbf{A} \mathbf{J}_{\mathbf{y}}\left(\widehat{\mathbf{x}}_{\lambda}\right)\right)-\sigma^{2}
$$

which depends solely on $\mathbf{y}$, thus, can be accessed in practice. Refer to Ref.(12) for the proof of the unbiasedness of UPRE. Here, $\mathbf{J}_{\mathbf{y}}\left(\widehat{\mathbf{x}}_{\lambda}\right) \in \mathbb{R}^{N \times M}$ is a Jacobian matrix defined as:

$$
\left[\mathbf{J}_{\mathbf{y}}\left(\widehat{\mathbf{x}}_{\lambda}\right)\right]_{n, m}=\frac{\partial\left(\widehat{\mathbf{x}}_{\lambda}\right)_{n}}{\partial y_{m}}
$$

Any estimate $\widehat{\mathbf{x}}_{\lambda}$ can be regarded as a linear or non-linear transformation of the observed data $\mathbf{y}$. Here, we make the dependence explicit. In particular, if $\widehat{\mathbf{x}}_{\lambda}$ is a linear estimate: $\widehat{\mathbf{x}}_{\lambda}=\mathbf{F y}$, then, $\mathbf{J}_{\mathbf{y}}\left(\widehat{\mathbf{x}}_{\lambda}\right)=\mathbf{F}$. For the non-linear sparse estimate $\widehat{\mathbf{x}}_{\lambda}$ considered here, the computation of $\mathbf{J}_{\mathbf{y}}\left(\widehat{x}_{\lambda}\right)$ becomes essential for the UPRE evaluation.

The UPRE has been widely used in the statistics and signal processing communities, as a principled and efficient way for parameter selection with a variety of linear and non-linear estimators ${ }^{(7,8,12,13)}$. In this paper, for the non-linear sparse estimate by a given IRLS iterate, we develop a recursive procedure for computing UPRE, which enables us to evaluate the (estimated) prediction error for the sparse recovery. In particular, we incorporate matrix splitting strategy ${ }^{(14)}$ into the IRLS algorithms, to make the computation of Jacobian matrix tractable. The optimal $\lambda$ is then identified by exhaustive search for minimum UPRE.

Notations: To keep consistency throughout the paper, we use lowercase letter to denote vector, and uppercase for matrix. We denote the entry index of vector or matrix by the subscripts $m, n$ and $l$. The iteration is indexed by $i, j$ and $k$.

\section{Recursive UPRE for IRLS algorithm}

\subsection{Basic scheme of IRLS}

To solve $\mathbf{P}$ with fixed $\lambda$, the IRLS algorithm updates the solution $\mathbf{x}^{(i)}$ by solving the following data-dependent equations $^{(3,6)}$ :

$$
\left(\mathbf{A}^{\mathrm{T}} \mathbf{A}+\lambda \mathbf{D}^{\mathrm{T}} \Gamma_{(i-1)}^{-1} \mathbf{D}\right) \mathbf{x}^{(i)}=\mathbf{A}^{\mathrm{T}} \mathbf{y}
$$

at $i$-th iteration, where $\Gamma_{(i-1)} \in \mathbb{R}^{L \times L}$ is a diagonal matrix and $\left[\Gamma_{(i-1)}\right]_{l, l}=\left|\left(\mathbf{D} \mathbf{x}^{(i-1)}\right)_{l}\right|=\left|u_{l}^{(i-1)}\right| \quad$ for $l=1,2, \ldots, L$.

Eq.(4) leads to the following update of $\mathbf{x}^{(i)}$ :

$$
\mathbf{x}^{(i)}=\left(\mathbf{A}^{\mathrm{T}} \mathbf{A}+\lambda \mathbf{D}^{\mathrm{T}} \Gamma_{(i-1)}^{-1} \mathbf{D}\right)^{-1} \mathbf{A}^{\mathrm{T}} \mathbf{y}
$$

By Eq.(3), UPRE of the $i$-th iterate $\mu^{(i)}=\mathbf{A} \mathbf{x}^{(i)}$ is:

$$
\operatorname{UPRE}\left(\mu^{(i)}\right)=\frac{1}{M}\left\|\mathbf{A} \mathbf{x}^{(i)}-\mathbf{y}\right\|_{2}^{2}+\frac{2 \sigma^{2}}{M} \operatorname{Tr}\left(\mathbf{A} \mathbf{J}_{\mathbf{y}}\left(\mathbf{x}^{(i)}\right)\right)-\sigma^{2}
$$

The UPRE computation requires to evaluate $\mathbf{J}_{\mathbf{y}}\left(\mathbf{x}^{(i)}\right)$, which, however, cannot be directly evaluate from Eq.(5), since $\mathbf{x}^{(i)}$ is NOT a linear transformation of $\mathbf{y}$.

\subsection{Matrix splitting to solve IRLS}

Instead of the closed-form solution (5), we apply the matrix-splitting (MS) scheme ${ }^{(14)}$ to solve Eq.(4), which, as we will see later, enables us to evaluate $\mathbf{J}_{\mathbf{y}}\left(\mathbf{x}^{(i)}\right)$ in a recursive manner. This method splits the matrix $\mathbf{A}^{\mathrm{T}} \mathbf{A}+\lambda \mathbf{D}^{\mathrm{T}} \Gamma_{(i-1)}^{-1} \mathbf{D}$ as:

$$
(\underbrace{\left(\mathbf{C}+\lambda \mathbf{D}^{\mathrm{T}} \Gamma_{(i-1)}^{-1} \mathbf{D}\right)}_{\mathbf{B}_{(i-1)}}-\underbrace{\left(\mathbf{C}-\mathbf{A}^{\mathrm{T}} \mathbf{A}\right)}_{\mathbf{E}}) \mathbf{x}^{(i)}=\mathbf{A}^{\mathrm{T}} \mathbf{y}
$$

where $\mathbf{x}^{(i)}$ is solved by iteration indexed on $j$ :

$$
\mathbf{x}^{(i, j+1)}=\mathbf{B}_{(i-1)}^{-1}\left(\mathbf{A}^{\mathrm{T}} \mathbf{y}+\mathbf{E} \mathbf{x}^{(i, j)}\right)
$$

The MS iteration (7) is convergent to the unique solution of Eq.(4) for any initial $\mathbf{x}^{(i, 0)}$, if and only if the spectral radius $\rho\left(\mathbf{B}_{(i-1)}^{-1} \mathbf{E}\right)<1^{(14)}$. To guarantee the convergence, we choose $\mathbf{C}=\mathbf{A}^{\mathrm{T}} \mathbf{A}+\alpha \mathbf{I}>\mathbf{A}^{\mathrm{T}} \mathbf{A} \quad$ with $\quad \alpha>0, \quad$ such that $\quad$ (1) $\mathbf{E}=\mathbf{C}-\mathbf{A}^{\mathrm{T}} \mathbf{A}=\alpha \mathbf{I}$ is positive definite; (2) $\mathbf{B}_{(i-1)}$ is symmetric, positive definite, and thus, invertible; (3) $\rho\left(\mathbf{B}_{(i-1)}^{-1} \mathbf{E}\right)<1$.

Notice that it is difficult to compute $\mathbf{B}_{(i-1)}^{-1}$. We now use matrix inversion lemma (MIL) to solve it:

$$
\mathbf{B}_{(i-1)}^{-1}=\left(\mathbf{C}+\lambda \mathbf{D}^{\mathrm{T}} \Gamma_{(i-1)}^{-1} \mathbf{D}\right)^{-1}=\mathbf{C}^{-1}-\mathbf{C}^{-1} \mathbf{D}^{\mathrm{T}} \mathbf{G}_{(i-1)}^{-1} \mathbf{D} \mathbf{C}^{-1}
$$

with $\mathbf{G}_{(i-1)}=\frac{1}{\lambda} \Gamma_{(i-1)}+\mathbf{D C}^{-1} \mathbf{D}^{\mathrm{T}} \in \mathbb{R}^{L \times L}$. Thus,

$$
\begin{aligned}
\mathbf{x}^{(i, j+1)} & =\underbrace{\mathbf{C}^{-1}\left(\mathbf{A}^{\mathrm{T}} \mathbf{y}+\mathbf{E} \mathbf{x}^{(i, j)}\right)}_{\mathbf{b}^{(i, j)} \in \mathbb{R}^{N}} \\
& -\mathbf{C}^{-1} \mathbf{D}^{\mathrm{T}} \underbrace{\mathbf{G}_{(i-1)}^{-1} \mathbf{D C} \mathbf{C}^{-1}\left(\mathbf{A}^{\mathrm{T}} \mathbf{y}+\mathbf{E} \mathbf{x}^{(i, j)}\right)}_{\mathbf{v}^{(i, j)} \in \mathbb{R}^{L}}
\end{aligned}
$$

where $\mathbf{v}^{(i, j)}$ is also solved by matrix splitting method:

$$
\overbrace{(\underbrace{\left(\rho \mathbf{I}+\frac{1}{\lambda} \Gamma_{(i-1)}\right)}_{\mathbf{P}_{(i-1)} \in \mathbb{R}^{L \times L}}-\underbrace{\left(\rho \mathbf{I}-\mathbf{D C}^{-1} \mathbf{D}^{\mathrm{T}}\right)}_{\mathbf{Q} \in \mathbb{R}^{L \times L}})}^{\mathbf{G}_{(i-1)}} \mathbf{v}^{(i, j)}=\underbrace{\mathbf{D} \underbrace{-1}\left(\mathbf{A}^{\mathrm{T}} \mathbf{y}+\mathbf{E} \mathbf{x}^{(i, j)}\right)}_{\mathbf{b}^{(i, j)}}
$$

where $\rho \mathbf{I}>\mathbf{D} \mathbf{C}^{-1} \mathbf{D}^{\mathrm{T}}$, with the following iterate indexed on $k$ :

$$
\mathbf{v}^{(i, j, k)}=\mathbf{P}_{(i-1)}^{-1}\left(\mathbf{Q} \mathbf{v}^{(i, j, k-1)}+\mathbf{D} \mathbf{b}^{(i, j)}\right)
$$

Similar to Eq.(7), Eq.(9) is also guaranteed to converge.

\subsection{Recursion of Jacobian matrix}

In this part, we will see that the computation of Jacobian 
matrix becomes tractable by the above IRLS-MS algorithm. Let us begin with the inner loop of iteration (9), which is rewritten here as $\mathbf{v}=\mathbf{P}^{-1} \mathbf{z}$, where $\mathbf{z}=\mathbf{Q} \mathbf{v}^{(i, j, k-1)}+\mathbf{D} \mathbf{b}^{(i, j)} \in \mathbb{R}^{L}$, the super- and sub-scripts are ignored for brevity. The Jacobian matrix $\mathbf{J}_{\mathbf{y}}(\mathbf{v})$ is derived as:

$$
\left[\mathbf{J}_{\mathbf{y}}(\mathbf{v})\right]_{l, m}=\sum_{s=1}^{L}\left[\mathbf{P}^{-1}\right]_{l, s} \frac{\partial z_{s}}{\partial y_{m}}+\sum_{s=1}^{L} z_{s} \frac{\partial\left[\mathbf{P}^{-1}\right]_{l, s}}{\partial y_{m}}
$$

for the $(l, m)$-th entry. The first term of Eq.(10) is:

$$
\sum_{s=1}^{L}\left[\mathbf{P}^{-1}\right]_{l, s} \frac{\partial z_{s}}{\partial y_{m}}=\left[\mathbf{P}^{-1} \mathbf{J}_{\mathbf{y}}(\mathbf{z})\right]_{l, m}
$$

where $\mathbf{J}_{\mathbf{y}}(\mathbf{z})=\mathbf{Q} \mathbf{J}_{\mathbf{y}}\left(\mathbf{v}^{(i, j, k-1)}\right)+\mathbf{D} \mathbf{J}_{\mathbf{y}}\left(\mathbf{b}^{(i, j)}\right), \quad$ by the property of Jacobian matrix. Noting that $\left[\mathbf{P}^{-1}\right]_{l, s}=0$, if $s \neq l$, the second term of (10) for fixed $l$ and $m$ is:

$$
\begin{aligned}
\sum_{s=1}^{L} z_{s} \frac{\partial\left[\mathbf{P}^{-1}\right]_{l, s}}{\partial y_{m}} & =\underbrace{-z_{l} \frac{1}{\lambda}\left(\rho+\frac{1}{\lambda}\left|u_{l}^{(i-1)}\right|\right)^{-2} \cdot \operatorname{sign}\left(u_{l}^{(i-1)}\right)}_{s_{l}^{(i-1)}} \cdot \frac{\partial u_{l}^{(i-1)}}{\partial y_{m}} \\
& =\left[\mathbf{S}_{(i-1)} \mathbf{D} \mathbf{J}_{\mathbf{y}}\left(\mathbf{x}^{(i-1)}\right)\right]_{l, m}
\end{aligned}
$$

Here, $\mathbf{S}_{(i-1)} \in \mathbb{R}^{L \times L}$ is diagonal with $\left[\mathbf{S}_{(i-1)}\right]_{l, l}=s_{l}^{(i-1)}$ for $l=1,2, \ldots, L$. Finally, Eq.(10) becomes:

$$
\begin{aligned}
\mathbf{J}_{\mathbf{y}}\left(\mathbf{v}^{(i, j, k)}\right) & =\mathbf{P}_{(i-1)}^{-1} \mathbf{Q} \mathbf{J}_{\mathbf{y}}\left(\mathbf{v}^{(i, j, k-1)}\right)+\mathbf{P}_{(i-1)}^{-1} \mathbf{D J}_{\mathbf{y}}\left(\mathbf{b}^{(i, j)}\right) \\
& +\mathbf{S}_{(i-1)} \mathbf{D} \mathbf{J}_{\mathbf{y}}\left(\mathbf{x}^{(i-1)}\right)
\end{aligned}
$$

which expresses the recursion of Jacobian matrix.

From (8), we obtain that other evolutions of Jacobian matrices are:

$$
\begin{cases}\mathbf{J}_{\mathbf{y}}\left(\mathbf{b}^{(i, j)}\right) & =\mathbf{C}^{-1} \mathbf{A}^{\mathrm{T}}+\mathbf{C}^{-1} \mathbf{E} \mathbf{J}_{\mathbf{y}}\left(\mathbf{x}^{(i, j)}\right) \\ \mathbf{J}_{\mathbf{y}}\left(\mathbf{x}^{(i, j+1)}\right)=\mathbf{J}_{\mathbf{y}}\left(\mathbf{b}^{(i, j)}\right)-\mathbf{C}^{-1} \mathbf{D}^{\mathrm{T}} \mathbf{J}_{\mathbf{y}}\left(\mathbf{v}^{(i, j)}\right)\end{cases}
$$

Thus, we can compute the UPRE during the IRLS iterations:

$$
\operatorname{UPRE}\left(\mu^{(i, j)}\right)=\frac{1}{M}\left\|\mathbf{A} \mathbf{x}^{(i, j)}-\mathbf{y}\right\|_{2}^{2}+\frac{2 \sigma^{2}}{M} \operatorname{Tr}\left(\mathbf{A} \mathbf{J}_{\mathbf{y}}\left(\mathbf{x}^{(i, j)}\right)\right)-\sigma^{2}
$$

\subsection{Short summary}

We summarize the proposed IRLS-MS-UPRE as Algorithm 1, which enables us to solve $\mathbf{P}$ with a prescribed value of $\lambda$, and simultaneously evaluate the UPRE during the IRLS iterations.

To find the optimal value of $\lambda$, an intuitive idea is to repeatedly implement Algorithm 1 for various tentative values of $\lambda$, then, the minimum UPRE indicates the optimal $\lambda$ (see Fig. 2 for example). This global search, depicted in Algorithm 2, has been frequently used ${ }^{(1,7,8)}$.
Algorithm 1: IRLS-MS-UPRE: UPRE evaluation for IRLS

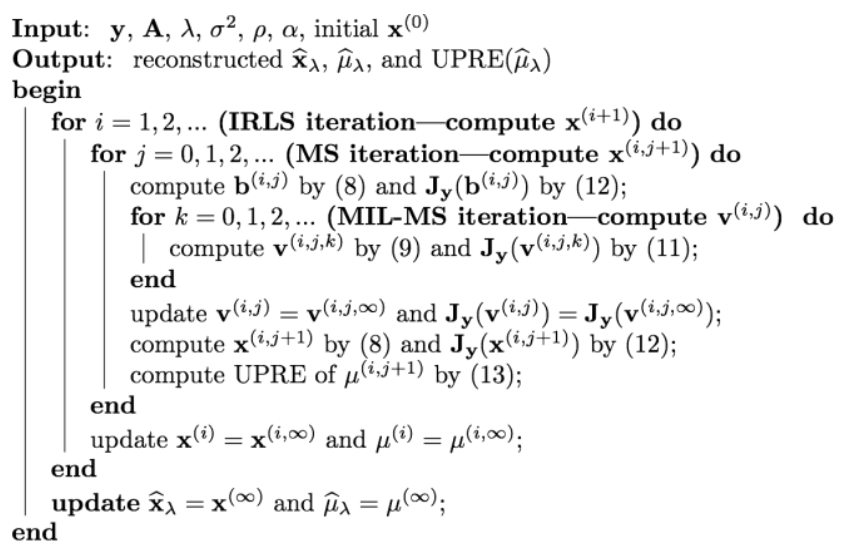

Algorithm 2: Global optimization of $\lambda$ for IRLS

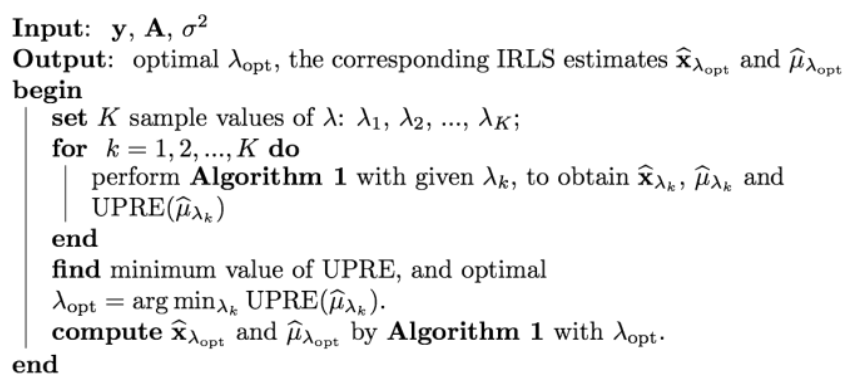

\section{Experimental results and discussions}

In this section, we are going to solve $\mathbf{P}$ by IRLS, and present the results by a few numerical examples.

The convergence of the algorithm does not necessarily lead to the minimum prediction error: it is possible that the best reconstruction quality can be achieved before convergence. In principle, we could optimize the number of iteration. However, in all our experiments of this paper, we implement the algorithm until the final convergence is reached, such that the reconstruction is indeed a solution to problem.

We assume that $\sigma^{2}$ (the variance of noise in $\mathbf{y}$ ) was known in all simulations to compute the UPRE. In practice, $\sigma$ can be estimated fairly reliably using, for example, MAD $\operatorname{method}^{(12)}$.

\subsection{Random example}

First, we consider a random example: we randomly generate the matrix $\mathbf{A} \in \mathbb{R}^{300 \times 500}$, and set $\mathbf{x} \in \mathbb{R}^{500}$ as a sparse vector with very few non-zeros (in this example, 10 non-zeros). Then, we add the noise $\varepsilon$ with noise variance $\sigma^{2}$ to obtain the observed data $\mathbf{y}=\mathbf{A x}+\boldsymbol{\varepsilon}$. Since $\mathbf{x}$ itself is already sparse in the original domain, for this case, we are 
solving a special case of $\mathbf{P}$ with decomposition matrix being identity ( $\mathbf{D}=\mathbf{I})$.

We apply Algorithm 1 to solve $\mathbf{P}$ with fixed $\lambda=1$. Fig.1-(1) shows that the objective value keeps decreasing to converge; Fig.1-(2) shows the evolutions of UPRE and true EPE during the iterations. We can see that the UPRE is always a reliable substitute for EPE.
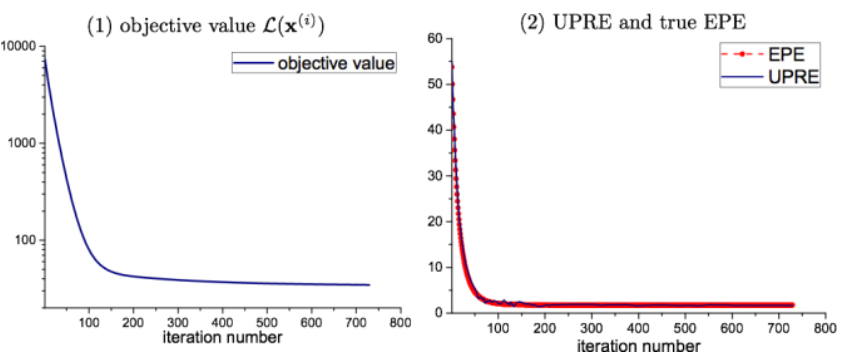

Figure 1 The convergence of IRLS-MS with $\lambda=1$.

For global search, we implement Algorithm 2 for 50 tentative values of $\lambda$, and obtain the corresponding UPRE. Fig. 2 shows the relation between UPRE and $\lambda$, where the minimum point indicates optimal value of $\lambda$.

UPRE as a function of $\lambda$ (global)

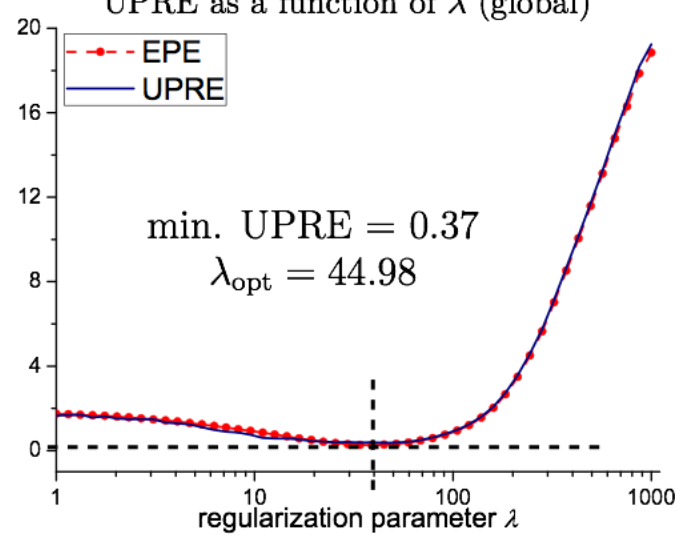

Figure 2 The relation between UPRE and $\lambda$.

Fig. 3 shows a part of the reconstructed $\mathbf{x}$, which is close to the true vector.

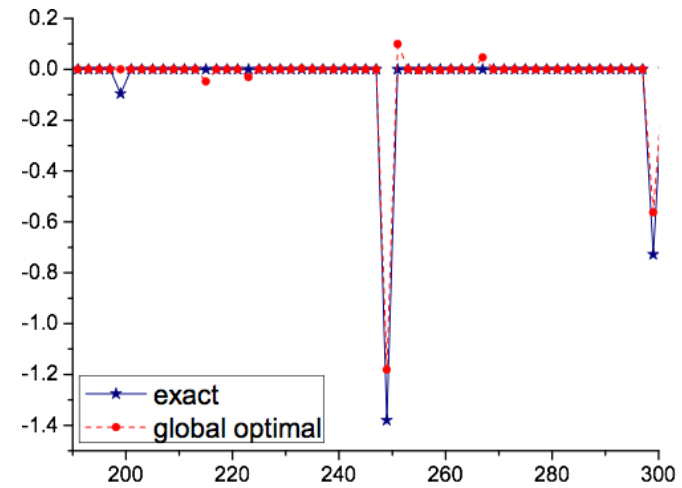

Figure 3 A part of the reconstruction of $\mathbf{x}$.

\subsection{Deconvolution of 1-D signal}

We now consider a typical 1-D signal $\mathbf{x}$. A represents a convolution matrix, which is circulant under periodic boundary condition, constructed from a Gaussian kernel. We use decimated wavelet transform (DWT) and choose Haar basis as the analysis matrix D. Fig.4 shows the relation between UPRE and $\lambda$.

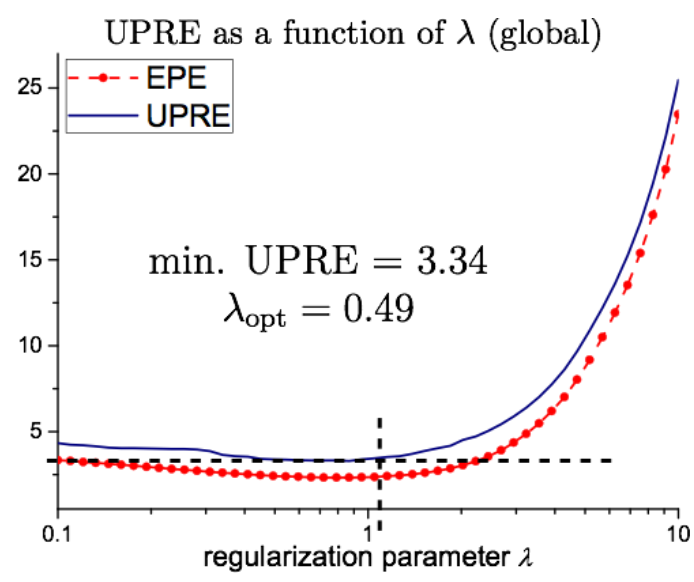

Figure 4 The relation between UPRE and $\lambda$.

Fig. 5 shows a part of the reconstructed $\mathbf{x}$, which is close to the true vector.

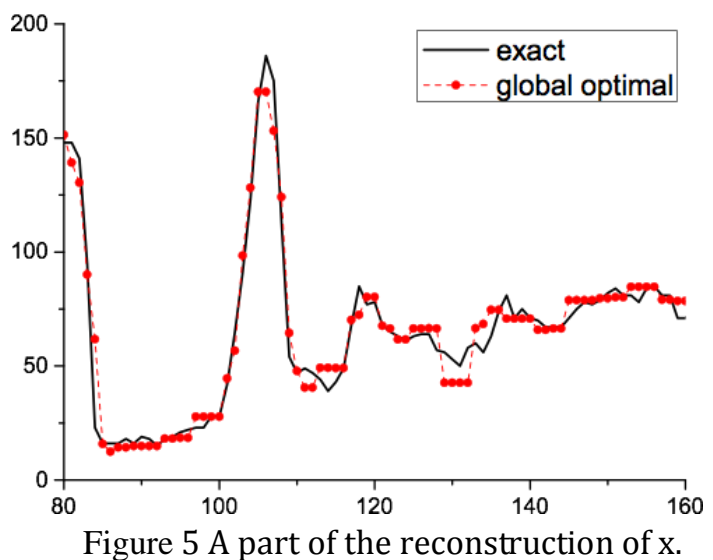

\section{Conclusions}

UPRE has been proven a powerful tool to select regularization parameter ${ }^{(7,8)}$. In this paper, to solve $\ell_{1}$-minimization problem, we proposed a recursive UPRE for IRLS algorithms, by incorporating matrix splitting scheme. It enables us to monitor the (estimated) prediction loss during the IRLS iterations, without referring to the true unknown data.

Theoretical derivations in this work related to the UPRE evaluation can be extended, in principle, to other types of 
regularizers and regularized iterative reconstruction algorithms. We would also like to emphasize that not limited to the simple example shown here, the developed recursive UPRE can be applied to many practical applications, e.g. compressed sensing ${ }^{(5)}$ and image deconvolution $^{(4,12)}$.

\section{Acknowledgment}

The work was supported by the National Natural Science Foundation of China under Grant No. 61401013. The authors are obliged to Prof. Thierry BLU and Hanjie PAN for many useful discussions on this article.

\section{References}

(1) Trevor Hastie, Robert Tibshirani, and Jerome Friedman, The Elements of Statistical Learning, Vol. 2, Springer, 2009

(2) Robert Tibshirani, "Regression shrinkage and selection via the lasso," Journal of the Royal Statistical Society. Series B (Methodological), pp. 267-288, 1996

(3) Hanjie Pan and Thierry Blu, "An iterative linear expansion of thresholds for $\ell_{1}$-based image restoration," IEEE Transactions on Image Processing, Vol. 22, No. 9, pp. 3715-3728, 2013

(4) Amir Beck and Marc Teboulle, "A fast iterative shrinkage-thresholding algorithm for linear inverse problems," SIAM Journal on Imaging Sciences, Vol. 2, No. 1, pp. 183-202, 2009

(5) Emmanuel J. Candes, Justin K. Romberg, and Terence Tao, "Stable signal recovery from incomplete and inaccurate measurements," Communications on Pure and Applied Mathematics, Vol. 59, No. 8, pp. 1207-1223, 2006

(6) Ingrid Daubechies, Ronald De Vore and Massimo Fornasier, "Iteratively reweighted least squares minimization for sparse recovery," Communications on Pure and Applied Mathematics, Vol. 63, No. 1, pp. 1-38, 2010

(7) C. Vonesch, S. Ramani, and M. Unser, "Recursive risk estimation for non-linear image deconvolution with a wavelet-domain sparsity constraint," in Proceedings of the 15th IEEE International Conference on Image Processing, San Diego CA, USA, October 12-15, 2008, pp. 665-668.

(8) R. Giryes, M. Elad, and Y.C. Eldar, "The projected GSURE for automatic parameter tuning in iterative shrinkage methods," Applied and Computational Harmonic Analysis, Vol. 30, No. 3, pp. 407-422, 2010

(9) V.A. Morozov, Methods for Solving Incorrectly Posed
Problems, Springer-Verlag, New York, 1984

(10) Per Christian Hansen, "Analysis of discrete ill-posed problems by means of the L-curve," SIAM Review, Vol. 34, No. 4, pp. 561-580, 1992

(11) G.H. Golub, M. Heath, and G. Wahba, "Generalized cross-validation as a method for choosing a good ridge parameter," Technometrics, Vol. 21, No. 2, pp. 215-223, 1979

(12) F. Xue and T. Blu, "A novel SURE-based criterion for parametric PSF estimation," IEEE Transactions on Image Processing, Vol. 24, No. 2, pp. 595-607, 2015

(13) Charles M Stein, "Estimation of the mean of a multivariate normal distribution," The Annals of Statistics, pp. 1135-1151, 1981

(14) Richard S. Varga, Matrix Iterative Analysis, Vol. 27, Springer Series in Computational Mathematics, 2000 